\title{
A REMARK ON RELATIVE HOMOLOGY AND COHOMOLOGY GROUPS OF A GROUP
}

\author{
To Zyoiti Suetuna on his 60th Birthday
}

\section{TADASI NAKAYAMA}

Let $G$ be a group and $H$ a subgroup of $G$. With a left $G$-module $M$, relative cohomology groups $H^{n}(G, H, M)$ of $G$ on $M$, relative to $H$, have been defined by Adamson [1] and may be expressed as $\operatorname{Ext}_{(G, H)}^{n}(Z, M)$ in the notation of relative homological algebra of Hochschild [2], where $Z$ denotes the $G$-module of rational integers (acted by $G$ trivially). Regarding $M$ as a right $G$-module, $\operatorname{Tor}_{n}^{(G, H)}(M, Z)$ are similarly relative homology groups $H_{n}(G, H, M)$. In case $H$ is of finite index in $G$, Hochschild [2] defines further negative-dimensional relative homology and cohomology groups. He then remarks that these complete relative homology and cohomology structures are separate (contrary to the absolute case $H=1$ ). Indeed he exhibits an example of $G, H, M$ (with $H$ even normal in $G$ ) such that $H^{n}(G, H, M)=0$ for every $n=0, \pm 1, \pm 2, \ldots$ and $H_{n}(G, H, M)$ is a group of order 2 for every $n=0, \pm 1, \pm 2, \ldots$ This, however, does not exclude the possibility that negative-dimensional relative homology groups $H_{-n}(G, H, M)$ are in close relationship with positive-dimensional relative cohomology groups on some $G$-module $N$ other than $M$. In fact, in case $H$ is a normal subgroup of $G$, we have $H_{-n}(G, H, M) \approx H_{-n}\left(G / H, M_{H}\right)$ $\approx H^{n-1}\left(G / H, M_{H}\right)$ (where $M_{H}$ denotes as usual the residue-module of $M$ with respect to the submodule generated by the elements of form $u-h u(u \in M$, $h \in H)$ and this is isomorphic to $H^{n-1}\left(G / H, N^{H}\right) \approx H^{n-1}(G, H, N)$ if $M_{H}$ is $G$-isomorphic to $N^{H}$ (where $N^{H}$ is the submodule of $N$ consisting of all elements of $N$ left invariant by $H^{\prime}$ ) ; this holds not only for $n>0$ but for all $n=0, \pm 1$, $\pm 2, \ldots$ Now we want to show that a similar phenomenon prevails also in case of a non-normal subgroup $H$.

Thus, let $H$ be a subgroup of finite index in a group $G$ and $K_{0}$ be the largest normal subgroup of $G$ contained in $H$, i.e. the intersection of all conjugates of $H$ in $G$. For $G$ modules $M$ and $N$, we consider the following condition:

Received September 7, 1959. 
(A) There exists a $G$-homomorphism $\kappa_{0}$ of $M$ into $N$ such that $\kappa_{0}$ induces a $(G-)$ isomorphism of $M_{K_{0}}$ onto $N^{K_{0}}$, and, moreover, we have $H^{-1}\left(K / K_{0}, M_{K_{0}}\right)=H^{0}\left(K / K_{0}, M_{K_{0}}\right)=0$ for every subgroup $K$ of $H$ which is an intersection of conjugates of $H$ in $G$.

This condition may also be formulated as follows:

$\left(A^{\prime}\right)$ There exists a $G$-homomorphism $\kappa_{0}$ of $M$ into $N$ such that $\kappa_{0}$ induces a (G-) isomorphism of $M_{K_{0}}$ onto $N^{K_{0}}$ and for every subgroup $K$ of $H$ which is an intersection of conjugates of $H$ in $G$ the homomorphism $\kappa(K)$ of $M$ into $N$ defined by

$$
\kappa(K) u=\sum_{K \ni \rho \bmod K_{0}} \rho \kappa_{0} u \quad(u \in M),
$$

$\rho$ running over a representative system of cosets of $K_{0}$ in $K$, induces an isomorphism of $M_{K}$ onto $N^{K}$.

Indeed, the endomorphism $v \rightarrow \sum_{K \ni \circ \bmod K_{0}} \rho v\left(v \in N^{K_{0}}\right)$ of $N^{K_{0}}$ induces a monomorphism (resp. an epimorphism) of $\left(N^{K_{0}}\right)_{\left(K / K_{0}\right)}$ to $\left(N^{K_{0}}\right)^{\left(K / K_{0}\right)}=N^{K}$ if and only if $H^{0}\left(K / K_{0}, N^{K_{0}}\right)=0$ (resp. $\left.H^{-1}\left(K / K_{0}, N^{K_{0}}\right)=0\right)$. Combining this consideration with $G$-isomorphism of $M_{K_{0}}$ and $N^{K_{0}}$ induced by $\kappa_{0}$, we see the equivalence of the conditions $(A),\left(A^{\prime}\right)$ readily.

We want also to note that if $(A)$ (or $\left(A^{\prime}\right)$ ) is the case and if $K$ is an intersection of non-void set of conjugates of $H$ in $G$, which is not necessarily a subgroup of $H$, the homomorphism of $M$ into $N$ defined by the same formula as (1) induces an isomorphism of $M_{K}$ onto $N^{K}$, as follows readily from the $G$ isomorphism property of $\kappa_{0}$ by an easy conjugation consideration. With this generalized significance of $\kappa(K)$, we observe also that if $K, L$ are two intersections of non-void sets of conjugates of $H$ in $G$ and if $L \supset K$ then

$$
\kappa(L)=\sum_{L \ni \rho, \bmod K} \rho \kappa(K),
$$

$\rho$ running over a representative system of right cosets of $K$ in $L$. We have also

$$
\kappa\left(\sigma K \sigma^{-1}\right)=\sigma \kappa(K) \sigma^{-1} \text {. }
$$

Now, with the condition $(A)$ (or $\left(A^{\prime}\right)$ ) we assert

Theorem. ${ }^{1)}$ Let $H$ be a subgroup of finite index in a group $G$. For $G$ -

1) The theorem will be applied in a subsequent paper to a study of fundamental exact sequences in homology and cohomology of finite groups. Cf. remark at the end. 
modules $M, N$, suppose that the condition $(A)$ (or, equivalently, $\left.\left(A^{\prime}\right)\right)$ is satisfied. Then

$$
H_{n}(G, H, M) \approx H^{-n-1}(G, H, N)
$$

for all $n \equiv 0$. More precisaly, if $\sum_{n \geqq 0} X_{n}$ is the standard complete complex $G$ relative to $H$, the complexes $\sum M \otimes_{G} X_{n}, \sum \operatorname{Hom}_{G}\left(X_{-n-1}, N\right)$ with respective differentiations $\partial, \delta$ are isomorphic by an isomorphism mapping $M \otimes_{G} X_{n}$ onto $\operatorname{Hom}_{G}\left(X_{-n-1}, N\right)$.

Proof. Relative homology and cohomology groups $H_{n}(G, H ; M), H^{n}(G, H$; $M$ ), with $H$ of finite index in $G$, are defined most conveniently by means of the standard complete complex of $(G, H)$, i.e. the exact sequence

$$
\cdots \rightarrow x_{1} \stackrel{\partial_{1}}{\longrightarrow} x_{0} \stackrel{\partial_{0}}{\longrightarrow} X_{-1} \stackrel{\partial_{-1}}{\longrightarrow} X_{-2} \stackrel{\partial_{-2}}{\longrightarrow} \cdots
$$

in which each $X_{n}$ with $n \geqq 0$ is the $G$-module having the totality of $(n+1)$ tuples $\left(\sigma_{0} H, \sigma_{1} H, \ldots, \sigma_{n} H\right)$ of right $H$-cosets in $G$ as $Z$-basis and the map $\partial_{n}: X_{n} \rightarrow X_{n-1}, n>0$, is defined by (linearity and)

$$
\partial_{n}\left(\sigma_{0} H, \ldots, \sigma_{n} H\right)=\sum_{i=0}^{n}(-1)^{i}\left(\sigma_{0} H, \ldots, \sigma_{i-1} H, \sigma_{i+1} H, \ldots, \sigma_{n} H\right)
$$

while $X_{-n}, n>0$, is the $G$-module $\operatorname{Hom}_{Z}\left(X_{n-1}, Z\right)$ dual to $X_{n-1}$ and the map $\partial_{-n}: X_{-n} \rightarrow X_{-n-1}, n \geqq 0$, is dual of $\partial_{n+1}$, and further, the map $\partial_{0}: X_{0} \rightarrow X_{1}$ is the combination of the coefficient sum homomorphism $X_{0} \rightarrow Z$ and its dual $Z \rightarrow X_{-1} ;$ all the modules $X_{n}, n \geqq 0$, are $(G, H)$-projective, all the maps $\partial_{n}$, $n \geqq 0$, are $G$-homomorphic, and the sequence is $(G, H)$-exact, in the sense of Hochschild [2]. We observe also that each $X_{n}$ is in fact $G$-isomorphic to its dual $X_{-n-1}$ (and is, hence, $(G, H)$-injective too); for $n \geqq 0$ the isomorphism is given by associating $\left(\sigma_{0} H, \ldots, \sigma_{n} H\right)$ with the element $\left\{\sigma_{0} H, \ldots, \sigma_{n} H\right\}$ of $\operatorname{Hom}_{Z}\left(X_{n}, Z\right)$ which maps $\left(\sigma_{0} H, \ldots, \sigma_{n} H\right)$ into 1 but other $(n+1)$-tuples to 0.

(Relative) cohomology groups $H^{n}(G, H ; N), n=$, on a $G$-module $N$ is defined by the sequence

$$
\begin{aligned}
\cdots & \stackrel{\partial}{\longleftarrow} \operatorname{Hom}_{G}\left(x_{1}, N\right) \stackrel{\partial}{\longleftarrow} \operatorname{Hom}_{G}\left(X_{0}, N\right) \stackrel{\partial}{\longleftarrow} \operatorname{Hom}_{G}\left(X_{-1}, N\right) \\
& \stackrel{\partial}{\longleftarrow} \operatorname{Hom}_{G}\left(X_{-2}, N\right) \stackrel{\partial}{\longleftarrow} \ldots .
\end{aligned}
$$

while (relative) homology groups $H_{n}(G, H ; M), n \equiv 0$, on a $G$-module $M$ is defined by the sequence 


$$
\ldots \stackrel{\partial}{\longrightarrow} M \otimes_{G} X_{1} \stackrel{\partial}{\longrightarrow} M \otimes_{G} X_{0} \stackrel{\partial}{\longrightarrow} M \otimes_{G} X_{-1} \stackrel{\partial}{\longrightarrow} M \otimes_{G} X_{-2} \cdots,
$$

both sequences being derived from (4) in natural manner. As $X_{-n-1}$ is $\operatorname{Hom}_{Z}\left(X_{n}, Z\right)$ (and is in fact isomorphic to $X_{n}$ itself) we have an $G$-isomorphism

$$
\nu: N \otimes_{z} X_{n} \approx \operatorname{Hom}_{Z}\left(X_{-n-1}, N\right)
$$

where $v \otimes x(v \in N, x \in X)$ in the left-hand side is mapped to the element $\nu(v \otimes x)$ of the right-hand side such that $\nu(v \otimes x) f=(f x) v$ for $f \in X_{-n-1}$ $=\operatorname{Hom}_{Z}(X, Z)$; the map is not only $(G$-)homomorphic but isomorphic since $X_{n}$ has an (independent) finite $Z$-basis. Hence we have an isomorphism, denoted also by $\nu$,

$$
\left(N \otimes_{z} X_{n}\right)^{G} \approx \operatorname{Hom}_{\theta}\left(X_{-n-1}, N\right) .
$$

Now, we first consider the case $n \geqq 0$. There is a system of $(n+1)$-tuples

$$
s=\left(\sigma_{0} H, \sigma_{1} H, \ldots, \sigma_{n} H\right), \quad s^{\prime}=\left(\sigma_{0}^{\prime} H, \sigma_{1}^{\prime} H, \ldots, \sigma_{n}^{\prime} H\right), \ldots
$$

such that for every $(n+1)$-tuple $t=\left(\tau_{0} H, \tau_{1} H, \ldots, \tau_{n} H\right)$ there is one, and only one, among them, say $\mathrm{s}^{(\mu)}$, from which the given $(n+1)$-typle $t$ is obtained by the operation of an element of $G$, thus $t=\tau s^{(\mu)}(\tau \in G)$; here $\tau \equiv \tau_{0} \sigma_{0}^{(\mu)^{-1}}$ r. $\bmod$ $\sigma_{0}^{(\mu)} H \sigma_{0}^{(\mu)^{-1}}, \equiv \tau_{1} \sigma_{1}^{(\mu)^{-1}}$ r. $\bmod \sigma_{1}^{(\mu)} H \sigma_{1}^{(\mu)^{-1}}, \ldots$, and $\tau$ is determined uniquely up to r. $\bmod \sigma_{0}^{(\mu)} H \sigma_{0}^{(\mu)^{-1}} \cap \sigma_{1}^{(\mu)} H \sigma_{1}^{(\mu)^{-1}} \cap \cdots \cap \sigma_{n}^{(\mu)^{-1}} H \sigma_{n}^{(\mu)^{-1}}$. We see readily that every element of the tensor product $M \otimes{ }_{G} X_{n}$ is expressed in a form

$$
u \otimes_{G} s+u^{\prime} \otimes_{G} s^{\prime}+\cdots \quad\left(u, u^{\prime}, \ldots \in M\right) .
$$

Here the classes in the residue-modules $M_{K}, M_{K^{\prime}}, \ldots$ of the elements $u, u^{\prime}, \ldots$, respectively, are uniquely determined, where we put

(10) $K=\sigma_{0} H \sigma_{0}^{-1} \cap \sigma_{1} H \sigma_{1}^{-1} \cap \cdots \cap \sigma_{n} H \sigma_{n}^{-1}, \quad K^{\prime}=\sigma_{0}^{\prime} H \sigma_{0}^{\prime-1} \cap \sigma_{1}^{\prime} H \sigma_{1}^{\prime-1} \cap \cdots \cap$ $\sigma_{n}^{\prime} H \sigma_{n}^{\prime-1}, \ldots$

for brevity.

Now, let $\kappa=\kappa(K), \kappa^{\prime}=\kappa\left(K^{\prime}\right), \ldots$ be the homomorphisms of $M$ into $N$ which are described in the condition $\left(A^{\prime}\right)$ of our theorem and thus in particular induce isomorphisms $M_{K} \approx N^{K^{\prime}}, M_{K^{\prime}} \approx N^{K^{\prime}}$, . . respectively. We associate with (9) the element

$$
\sum_{\rho \text { r.mod } K} \rho(\kappa u) \otimes \rho s+\sum_{\rho^{\prime}, \bmod K^{\prime}} \rho^{\prime}\left(\kappa^{\prime} u^{\prime}\right) \otimes \rho^{\prime} s^{\prime}+\cdots
$$

of $N \otimes_{z} X_{n}$, where in the first sum $\rho$ runs over a representative system of right 
cosets modulo $K$ in $G$, in the second sum $\rho^{\prime}$ runs over a representative system of right cosets modulo $K^{\prime}$ in $G$, and so on. This element (11) of $N \otimes_{z} X_{n}$ is independent of the choices of these representative systems of cosets, as follows from the definitions of $\kappa, K$, etc., and is, moreover, determined uniquely by the element (9), irrespective of the special choices of elements $u, u^{\prime}, \ldots$ from their classes in $M_{K}, M_{K^{\prime}}, \ldots{ }^{2)}$ The former of the last remarks entails that (11) belongs in fact to $\left(N \otimes_{z} X_{n}\right)^{a}$. Thus, by $(9) \rightarrow(11)$ we obtain a homomorphism

$$
\varphi: M \otimes_{a} X_{n} \rightarrow\left(N \otimes{ }_{z} X_{n}\right)^{a} .
$$

We contend that this homomorphism is an isomorphism. Thus, suppose that (11) is 0 . Since $\{\rho s\},\left\{\rho^{\prime} s^{\prime}\right\}, \ldots$ are altogether the set of distinct $(n+1)$-tuples, this implies that each single term in the sum (11) is 0 , i.e. $\rho(\kappa u)=0$ for every $\rho$ r. $\bmod K$, etc. It follows then that $u, u^{\prime}, \ldots$ belong to the kernels of $\kappa, \kappa^{\prime}$, $\ldots$, i.e. to the 0 -classes in $M_{K}, M_{K^{\prime}}, \ldots$, respectively. This implies however that the element (9) is 0 , showing that $\varphi$ is monomorphic.

To prove further that $\varphi$ is epimorphic, consider any element of $\left(N \otimes_{z} X_{n}\right)^{G}$, which we can express in a form

$$
\sum_{\rho \text { r. mod } K} v^{(\rho)} \otimes \rho s+\sum_{\rho^{\prime} \text { r.mod } K} v^{\left(\rho^{\prime}\right)} \otimes \rho^{\prime} s^{\prime}+\cdots
$$

$\left(v^{(p)}, v^{\left(p^{\prime}\right)}, \ldots \in N\right)$; we assume that the unit element 1 appears in each of the representative systems $\{\rho\},\left\{\rho^{\prime}\right\}, \ldots$ From its invariance by the elements of $K$ we deduce that $v^{(1)}$ belongs to $N^{K}$. Its invariance by $\rho$ implies $v^{(\rho)}=\mu v^{(1)}$. Similarly we have $v^{(1)} \in N^{K^{\prime}}, v^{\left(\rho^{\prime}\right)}=\rho^{\prime} v^{(1)}$, etc. The elements $v^{(1)}, v^{\prime(1)}, \ldots$ of $N^{K}, N^{K^{\prime}}, \ldots$ may be expressed as $\kappa u, \kappa^{\prime} u^{\prime}, \ldots$ with $u, u^{\prime}, \ldots \in M$. So the element (12) assumes a form (11) and is contained in the image of $M \otimes_{a} X_{n}$ by $\varphi$. This proves that $\varphi$ is an isomorphism.

In case $n<0$ we consider $\left\{\sigma_{0} H, \ldots, \sigma_{m} H\right\}(m=-n-1)$ in place of $\left(\sigma_{0} H, \ldots\right)$ and obtain similarly an isomorphism $\varphi: M \otimes{ }_{G} X_{n} \approx\left(N \otimes X_{n}\right)^{G}$.

Before proceeding further, we observe that in deriving (11) from (9), to define $\varphi$, we need not assume that $s, s^{\prime}, \ldots$ in (9) are the specific $(n+1)$ tuples in (8). Thus we consider (9) to be an arbitrary expression of a given element of $M \otimes_{z} X_{n}$, in which $s, s^{\prime}, \ldots$ are allowed to be any $(n+1)$-tuples of right cosets of $H$ in $G$, and show that the element (11) is determined uniquely

2) We shall soon observe that the element (9) is independent of the choice of $s, s^{\prime}$, .... Indeed it is determined by the element (9) itseli, irrespective of its special form. 
by the given element (9) itself, independent of its particular form (9). Indeed, since any other similar expression for the same element (9) is devived from (9) by addition of differences like $u \otimes_{G} s-\sigma u \otimes_{G} \sigma s$ (up to trivial rules like cancelling equal terms with opposite signatures), we have only to prove the equality $\sum_{\rho \text { r.mod } K} \rho(\kappa u) \otimes \rho s=\sum_{\rho_{1}, \bmod K_{1}} \rho_{1}\left(\kappa_{1} \sigma u\right) \otimes \rho_{1} \sigma s$, where $K=\sigma_{0} H \sigma_{0}^{-1} \cap \cdots \cap \rho_{n} H_{\rho_{n}^{-1}}$ $\left(s=\left(\sigma_{0} H, \ldots, \sigma_{n} H\right)\right), K_{1}=\sigma H \sigma^{-1}, \kappa=\kappa(K), \kappa_{1}=\kappa(K)$. But this is certainly the case since $\kappa_{1}=\kappa\left(\sigma K_{\sigma}^{-1}\right)=\sigma \kappa(K) \sigma^{-1}$, as was observed before, and $\rho_{1} \sigma$ runs over a representative system of right cosets modulo $K$, in $G$, when $\rho_{1}$ runs over a such of right cosets modulo $K_{1}=\sigma K \sigma^{-1}$. A similar remark holds also in case $n<0$.

Now we consider the diagram

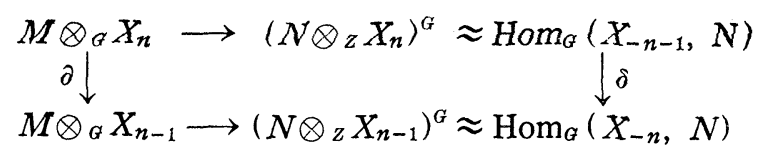

where the horizontal arrows are the isomorphisms $\varphi$ defined above.

We contend that the diagram is commutative. First consider the case $n \geqq 1$, and consider an element $\boldsymbol{u} \otimes_{G} s$ of $M \otimes_{a} X\left(s=\left(\sigma_{0} H, \ldots, \sigma_{n} H\right)\right)$. We have

$$
\partial\left(u \otimes_{G} s\right)=\sum_{i=0}^{n}(-1)^{i} u \otimes_{G}\left(\sigma_{0} H, \ldots, \sigma_{i-1} H, \sigma_{i+1} H, \ldots, \sigma_{n} H\right) \in M \otimes_{G} X_{n-1} .
$$

By our above remark about $\varphi$, we have

$$
\varphi\left(\partial\left(\boldsymbol{u} \otimes{ }_{G} s\right)\right)=\sum_{t=0}^{n}(-1)^{i} \sum_{\rho \mathrm{r}, \bmod K_{i}} \rho\left(\kappa_{i} u\right) \otimes \rho\left(\sigma_{0} H, \ldots, \sigma_{i-1} H, \sigma_{i+1} H, \ldots, \sigma_{n} H\right)
$$

where $K_{i}=K\left(\sigma_{0}, \ldots, \sigma_{i-1}, \sigma_{i+1}, \ldots, \sigma_{n}\right)=\sigma_{0} H \sigma_{0}^{-1} \cap \ldots \cap \sigma_{i-1} H \sigma_{i-1}^{-1} \cap \sigma_{i+1} H \sigma_{i+1}^{-1}$ $\cap \cdots \cap \sigma_{n} H \sigma_{n}^{-1}$ and $\kappa_{i}=\kappa\left(K_{i}\right)$. This element $\varphi\left(\partial\left(u \otimes{ }_{G} s\right)\right)$ of $\left(N \otimes{ }_{z} X_{n-1}\right)^{G}$ is associated by $\nu,(7)$, with the element of $\operatorname{Hom}_{G}\left(X_{-n}, N\right)$ mapping $\left\{\tau_{1} H, \ldots\right.$, $\left.\tau_{n} H\right\} \in X_{-n}=\operatorname{Hom}_{Z}\left(X_{n-1}, Z\right)$ to

$$
\sum_{i=0}^{n}(-1)^{i} \sum_{\rho, \bmod K_{i}} \delta_{\left(\tau_{1} H, \ldots, \tau_{l} H\right),\left(\rho \sigma_{0} H, \ldots, \rho \sigma_{i-1} H, \rho \sigma_{i} \iota_{1} H, \ldots, \rho \sigma_{u} H\right)} \rho\left(\kappa_{i} u\right) \in N
$$

(Kronecker $\delta^{\prime} s$ ). On the other hand, we have

$$
\varphi\left(\boldsymbol{u} \otimes{ }_{a} \boldsymbol{s}\right)=\sum_{\rho \operatorname{romad} K} \rho(\kappa u) \otimes \rho\left(\sigma_{0} H, \sigma_{1} H, \ldots, \sigma_{n} H\right),
$$

which corresponds by $\nu$ to the element of $\operatorname{Hom}_{G}\left(X_{-n-1}, N\right)$ mapping $\left\{\tau_{0} H, \tau_{1} H\right.$,

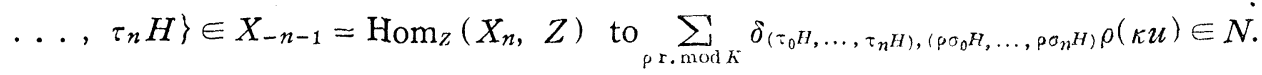


Its image by the coboundary operation $\delta$ is the element of $\operatorname{Hom}_{G}\left(X_{-n}, N\right)$ which maps $\left\{\tau_{1} H, \ldots, \tau_{n} H\right\} \in X_{-n}=\operatorname{Hom}_{Z}\left(X_{n-1}, Z\right)$ to

$$
\sum_{\rho \mathrm{r} . \bmod K} \sum_{i=0}^{n}(-1)^{i} \sum_{\sigma \mathrm{r} . \bmod H} \boldsymbol{\delta}_{\left\{\tau_{1} H, \ldots, \tau_{i} H, \sigma H, \tau_{i+1} H, \ldots, \tau_{n} H\right\},\left\langle\rho \sigma_{0} H, \ldots, \rho \sigma_{n} H\right\}} \rho(\kappa u) \in N,
$$

since $\partial\left\{\tau_{1} H, \ldots, \tau_{n} H\right\}=\sum_{i=0}^{n}(-1)^{i} \sum_{\rho \operatorname{r.mod} H}\left\{\tau_{1} H, \ldots, \tau_{i} H, \sigma H, \tau_{i+1} H, \ldots, \tau_{n} H\right\}$. In the summation $\sum_{\sigma \mathrm{r}, \bmod H}$ for fixed $\rho, i$ in (15) the only effective term is the one with $\sigma H=\rho \sigma_{i} H$, and (15) may be rewritten as

$$
\sum_{\rho} \sum_{\mathbf{r}, \bmod K} \sum_{i=0}^{n}(-1)^{i} \delta_{\left(\tau_{1} H, \ldots, \tau_{u} H\right),\left(\rho \sigma_{0} H, \ldots, \rho \sigma_{i-1} H, \rho \sigma_{i+1} H, \ldots, \rho \sigma_{n} H\right)} \rho(\kappa u) .
$$

Now, for each $i$ we have $K_{i} \supset K$ and

$$
\sum_{K_{i} \ni \rho, \bmod K} \rho(\kappa u)=\kappa_{i} u
$$

by (2). Thus (14) is in fact equal to (16) whence to (15), which shows that the diagram (13) is commutative.

Next consider the case $n<0$. Set $m=-n \geqq 1$. With $s=\left\{\sigma_{1} H, \ldots, \sigma_{m} H\right\}$ we have

$$
\begin{aligned}
& \partial\left(u \otimes \otimes_{G} s\right)=\sum_{i=0}^{m}(-1)^{i} u \otimes_{G} \sum_{\sigma \mathrm{r}, \bmod H}\left\{\sigma_{1} H, \ldots, \sigma_{i} H, \sigma H, \sigma_{i+1} H, \ldots, \sigma_{m} H\right\}, \\
& \varphi\left(\partial\left(u \otimes_{G} s\right)\right)=\sum_{i=0}^{m}(-1)^{i} \sum_{\sigma \mathrm{r} . \bmod H} \sum_{\rho \mathrm{r} . \bmod K_{\sigma}} \rho\left(\kappa_{\sigma} u\right) \\
& \otimes \rho\left\{\sigma_{1} H, \ldots, \sigma_{i} H, \sigma H, \sigma_{i+1} H, \ldots, \sigma_{m} H\right\}
\end{aligned}
$$

where $\kappa_{\sigma}=\sigma H \sigma^{-1} \cap \sigma_{1} H \sigma_{1}^{-1} \cap \cdots \cap \sigma_{m} H \sigma_{m}^{-1}$ and $\kappa_{\sigma}=\kappa\left(K_{s}\right)$. Thus $\varphi\left(\partial\left(u \otimes_{G} s\right)\right)$ is associated, by $\nu$, with the element of $\operatorname{Hom}_{G}\left(X_{m}, N\right)$ mapping $\left(\tau_{0} H, \tau_{1} H, \ldots\right.$, $\tau_{m} H$ ) to

$$
\sum_{i=0}^{m}(-1)^{i} \sum_{\sigma \mathrm{r} . \bmod H} \sum_{\rho \mathrm{r} . \operatorname{niod} K_{\sigma}} \delta_{\left(\tau_{0} H, \ldots, \tau_{m} H\right),\left(\rho \sigma_{1} H, \ldots, \rho \sigma H, \rho \sigma_{i+1} H, \ldots, \rho \sigma_{m} H\right) \rho\left(\kappa_{\sigma} u\right) \in N .}
$$

In the summation $\sum_{\rho \mathrm{r} . \bmod K_{\sigma}}$ for fixed $i, \sigma$ in (17) the only effective terms are the ones with $\rho_{\sigma} H=\tau_{i} H$. Hence (17) may be rewritten as

$$
\begin{aligned}
& \sum_{i=0}^{m}(-1)^{i} \sum_{\sigma \mathrm{r} . \bmod H} \sum_{\tau_{i} H \sigma^{-1} \ni \rho \text { r.mod } K_{\sigma}} \delta_{\left(\tau_{0} H, \ldots, \tau_{i-1} H, \tau_{i+1} H, \ldots, \tau_{m} H\right),\left(\rho \sigma_{1} H, \ldots, \rho \tau_{m} H\right) \rho\left(\kappa_{\sigma} u\right)} \\
& \quad=\sum_{i=0}^{m} \sum_{\rho} \text { r. } \sum_{\bmod K_{\sigma}} \delta_{\left(\tau_{0} H, \ldots, \tau_{i-1} H, \tau_{i+1} H, \ldots, \tau_{m} H\right),\left(\rho \tau_{1} H, \ldots, \rho \sigma_{m} H\right)} \rho\left(\kappa_{\sigma} u\right) .
\end{aligned}
$$

On the other hand, we have, with $K=\sigma_{1} H \sigma_{1}^{-1} \cap \cdots \cap \sigma_{m} H \sigma_{m}^{-1}$, 


$$
\varphi\left(u \otimes{ }_{a} s\right)=\sum_{\rho, \text { maod } \boldsymbol{E}} \rho(\kappa u) \otimes \rho\left\{\sigma_{1} H, \ldots, \sigma_{m} H\right\}
$$

and this corresponds by $\nu$ to the element of $\operatorname{Hom}_{\theta}\left(X_{m-1}, N\right)$ mapping $\left(\tau_{1} H\right.$, $\left.\ldots, \tau_{m} H\right)$ to

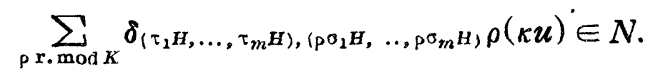

Hence $\delta\left(\nu\left(\varphi\left(u \otimes_{G} s\right)\right)\right)$ is the element of $\operatorname{Hom}_{G}\left(X_{m}, N\right)$ mapping $\left(\tau_{0} H, \tau_{1} H\right.$, $\left.\ldots, \tau_{m} H\right)$ to

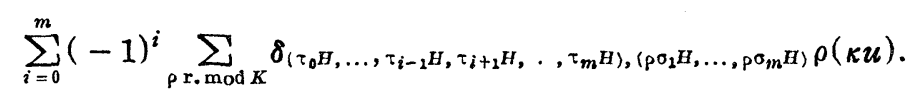

But, since $\kappa u=\sum_{K \ni \rho} \sum_{r, \bmod K_{\sigma}} \rho\left(\kappa_{\sigma} u\right)$, for each $\sigma$, the right-hand side of (18) coincides with (19). This proves the commutativity of (13) for $n<0$.

We finally consider the case $n=0$. For $s=\left(\sigma_{0} H\right)$ we have

$$
\begin{aligned}
& \partial\left(u \otimes_{G} s\right)=\partial\left(u \otimes_{G}\left(\sigma_{0} H\right)\right)=u \otimes_{G} \sum_{\sigma \cdot \bmod H}\left\{\sigma_{\sigma} \sigma H\right\}=u \otimes_{\sigma} \sum_{\sigma \cdot \bmod H}\{\sigma H\} \\
& \quad=\sum_{\sigma r \cdot \bmod H} \sigma^{-1} u \otimes_{G}\{H\}, \\
& \varphi\left(\partial\left(u \otimes_{G} s\right)\right)=\sum_{\sigma \text { r. niod } H} \sum_{\rho \text { r.mod } H} \rho\left(\kappa \sigma^{-1} u\right) \otimes \rho\{H\}
\end{aligned}
$$

with $\kappa=\kappa(H)$. Hence $\nu \varphi\left(\partial\left(u \otimes_{G} s\right)\right)$ is the element of $\operatorname{Hom}_{G}\left(X_{0}, N\right)$ which maps $(H) \in X_{0}$ onto

$$
\sum_{\sigma \mathbf{r} \cdot \bmod H} \sum_{\rho \mathrm{r} \cdot \bmod H}\{\rho H\}(H) \rho\left(\kappa \sigma^{-1} u\right)=\sum_{\sigma \mathrm{r} \cdot \bmod H} \kappa \sigma^{-1} u .
$$

On the other hand, we have

$$
\varphi\left(u \otimes_{G} s\right)=\varphi\left(u \otimes_{G}\left(\sigma_{0} H\right)\right)=\varphi\left(\sigma_{0}^{-1} u \otimes_{G}(H)\right)=\sum_{\rho \operatorname{r.mod} H} \rho\left(\kappa \sigma_{0}^{-1} u\right) \otimes \rho(H),
$$

and $\nu \varphi\left(u \otimes_{G} s\right)$ is the element of $\operatorname{Hom}_{G}\left(X_{-1}, N\right)$ mapping $\{\tau H\} \in X_{-1}$ onto $\sum_{\rho \operatorname{rrmod} H}\{\tau H\}(\rho H) \rho\left(\kappa \sigma_{0}^{-1} u\right) \tau\left(\kappa \sigma_{0}^{-1} u\right)$. Hence $\delta \nu \varphi\left(u \otimes_{G} s\right)$ is the element of $\operatorname{Hom}_{G}\left(X_{0}, N\right)$ mapping $(H)$ onto

$$
\sum_{\tau \mathrm{r} . \bmod H} \tau\left(\kappa \sigma_{0}^{-1} u\right)
$$

Here, since $\kappa=\sum_{H \ni p \bmod K_{0}} \rho \kappa_{0}$ with $\kappa_{0}=\kappa(K), K_{0}$ being the intersection of all conjugates of $H$ in $G$, the sum (21) is equal to $\sum_{\sigma r . \bmod K_{0}} \sigma \kappa_{0} \sigma_{0}^{-1} u$, and this is in turn equal to $\sum_{\sigma \text { r. } \bmod K_{0}} \sigma \sigma_{0}^{-1} \kappa_{0} u=\sum_{\sigma \text { romod } K_{0}} \sigma \kappa_{0} u$, as $\kappa_{0}$ is a $G$-homomorphism. Similarly the righthand side of $(20)$ is equal to $\sum_{\sigma \operatorname{romod} H} \sum_{H \ni P \bmod K_{0}} \kappa_{0} \rho \sigma u^{-1}=\sum_{\sigma \bmod K_{0}} \kappa_{0} \sigma u=\sum_{\sigma \bmod K_{0}} \sigma \kappa_{0} u$ : 
Thus (20) and (21) are equal and this proves the commutativity of (13) for $n=0$.

The isomorphism of $\varphi$ and the commutativity of (3) shows that the complexes $\sum M \otimes_{G} X_{n}, \sum \operatorname{Hom}_{G}\left(X_{-n-1}, N\right)$ with differentiations $\partial, \delta$ are isomorphic, as was asserted.

Remark. If $H$ is (not only of finite index in $G$ but) of finite order and if $H^{-1}(K, M)=H^{0}(K, M)=0$ for every subgroup $K$ of $H$ which is an intersection of conjugates of $H$ in $K$, then the condition $(A)$ (or equivalently $\left(A^{\prime}\right)$ ) is satisfied with $N=M$. For, we have then in particular $H^{-1}\left(K_{0}, M\right)=H^{0}\left(K_{0}, M\right)=0$, which implies that the $G$-endomorphism $\kappa_{0}$ of $M$ defined by $\kappa_{0} u=\sum_{\rho \in K_{0}} \rho u$ induces an isomorphism of $M_{K_{0}}$ onto $M^{K_{0}}$, and furthermore $\kappa(K)$ defined by (1) with our $\kappa_{0}$, just defined, induces an isomorphism of $M_{K}$ onto $M^{K}$, because of $H^{-1}(K, M)=H^{0}(K, M)=0$, for each $K$.

The application alluded to in the foot-note 1) will be under this stronger assumption.

\section{REFERENCES}

[1] I. T. Adamson, Cohomology theory for non-normal subgroups and non-normal fields, Proc. Glasgow Math. Assoc. 2 (1954-6), 67-76.

[2] G. Hochschild, Relative homological algebra, Trans. Amer. Math. Soc. 82 (1956), 246269.

Nagoya University 